\title{
Review of ultrasensitive readout for micro-/nanofluidic devices by thermal lens microscopy
}

\author{
Chihchen Chen $\odot{ }^{\text {a, } *}$ Hisashi Shimizu $\odot,{ }^{b}$ and Takehiko Kitamori ${ }^{\mathrm{a}, \mathrm{b}}$ \\ ${ }^{a}$ National Tsing Hua University, Institute of Nanoengineering and Microsystems, \\ Department of Power Mechanical Engineering, Hsinchu, Taiwan \\ ${ }^{\mathrm{b}}$ The University of Tokyo, Research Organization of Micro-Nano Multi Functional Device, \\ Department of Mechanical Engineering, Bunkyo, Tokyo, Japan
}

\begin{abstract}
Thermal lens microscopy (TLM) utilizes the effects, such as changes in the refractive index, caused by heat generated in the sample for the detection of nonfluorescent analytes with at least a hundred-fold enhancement in sensitivity compared with optical absorbance spectrometry. Micro-/nanofluidic devices can provide specificity and sample manipulation capabilities. The integration of these two technologies exposes the potential to attain the holy grail of continuous, real-time, label-free, specific, and ultrasensitive detection, which find applications in environmental monitoring, quality control of chemical manufacturing, single-cell analysis, and biomedicines. Here, we summarize the recent advances in the instrument development and innovative applications, and suggest future directions of research of TLM. () The Authors. Published by SPIE under a Creative Commons Attribution 4.0 Unported License. Distribution or reproduction of this work in whole or in part requires full attribution of the original publication, including its DOI. [DOI: 10.1117/1 .JOM.1.2.020901]
\end{abstract}

Keywords: thermal lens microscopy; microfluidics; nanofluidics; photothermal spectroscopy; thermal lens spectrometry; gradient-index lens.

Paper 20017V received Oct. 12, 2020; accepted for publication Feb. 15, 2021; published online Mar. 9, 2021.

\section{Introduction}

Thermal lens microscopy (TLM) is one of the photothermal techniques, which are extremely sensitive, versatile, and robust, permitting the measurement of absorbance and photothermal properties of the irradiated sample. ${ }^{1,2}$ Photothermal techniques measure the effect (in the form of heat or vibration), resulting from the optical absorption in a sample rather than measuring absorption directly. Therefore, much greater sensitivity can be attained because a signal from a small or zero baseline is measured. In contrast, most techniques that measure absorbance, such as ultraviolet-visible (UV/VIS) spectrophotometry, aim to discern small differences caused by the dilute sample plus all the scattering losses in a large signal, in which sensitivity is limited by the availability of a stable and noise-free reference signal.

Since the discovery of the thermal lens effect by Gordon et al., ${ }^{3}$ photothermal techniques have developed in analytical chemistry from the 1970s. ${ }^{4,5}$ In the ' 80 s and '90s, high sensitivity and ultrafast spectroscopy were especially highlighted, respectively, along with the progress of laser light sources. ${ }^{6,7}$ In the late '90s, Uchiyama et al. ${ }^{8}$ has realized photothermal measurements under a microscope for the first time and called it TLM. Then, TLM became established as a detection technique for microfluidic chips from around the 2000s. The detailed history of photothermal spectroscopy is described in another book. ${ }^{9}$ This review focuses on the TLM readout for microfluidics and nanofluidics, and introduces the basic concept of the thermal lens spectroscopy for ultrahigh sensitive measurements of optical absorption in contrast to fluorescence techniques that are widely used in microfluidic and nanofluidic applications.

Despite their excellent sensitivity, however, photothermal techniques are in general less userfriendly due to the capital/maintenance cost, complexity in the optical setup, and vulnerability to errors because of the fundamental dependence on a multitude of parameters involved. For instance, the thermal conductivity of the solvent must be carefully evaluated for determining

*Address all correspondence to Chihchen Chen, chihchen@mx.nthu.edu.tw 
the absorbance with high accuracy. As a result, the concentration of the analyte is often determined by establishing a calibration curve under the same experimental conditions, which is cumbersome and sometimes infeasible. Clearly, for analytical purposes, the enhanced performance of photothermal techniques is required to justify the increase in cost to compete with alternative techniques, e.g., electrochemical detection, that might achieve a comparable detection power. ${ }^{10-14}$ Compared with other photothermal techniques, such as optoacoustic spectroscopy, thermal lens spectrometry (TLS), and interferometric techniques, TLM utilizes tightly focused lasers and a microscopic observation system, which is indispensable for microfluidic applications. In addition, coaxially introduced lasers offer robust and simplified optics suitable for miniaturization. ${ }^{15-19}$ The advantages of miniaturization include not only much-reduced instrument cost, but also greater compatibility with micro-/nanofluidic devices that can equip the ultrahigh sensitivity provided by $\mathrm{TLM}^{20,21}$ with functions such as sample pretreatment, ${ }^{22}$ fractionation, ${ }^{23}$ parallel processing, ${ }^{24,25}$ sequential fluid handling, ${ }^{26,27}$ and microenvironment regulation. ${ }^{28}$ Hence, a highly sought system of high specificity, sensitivity, throughput with enhanced temporal and spatial resolution may be formed by integrating TLM and micro-/ nanofluidics. Here, we highlight recent endeavors toward this goal and relevant emerging novel applications, with the particulate focus on the detection of analytes in liquids. For applications of TLM or TLS to gas or solid samples, we refer the reader to these excellent reviews. ${ }^{29,30}$ Other advances on photothermal microscopy such as imaging of nanoparticles and cells are summarized in another recent review. ${ }^{31}$

\section{TLM at Microscale}

\subsection{Photothermal Effect}

Photothermal effect is relevant to optical absorption followed by nonradiative relaxation of molecules. The electronic transitions for absorption, fluorescence, and nonradiative relaxation are illustrated in a Jablonski diagram shown in Fig. 1(a). An electron in the ground state is excited by a photon and relaxes through radiative or nonradiative relaxation. The probability of the radiative relaxation process is the so-called fluorescence quantum yield $\left(\phi_{f}\right)$. However, almost all kinds of molecules have extremely low fluorescence quantum yields which are nearly zero. Even for fluorescence molecules, the quantum yields are 0.5 or less except for some dye molecules such as fluorescein and rhodamine. Therefore, all molecules practically experience non-radiative relaxation following absorption, which guarantees the applicability of photothermal spectroscopy equal to absorption spectrophotometry. Moreover, the sensitivity of photothermal spectroscopy is easily amplified by increasing the intensity of light (e.g., using a focused laser) similar to fluorescence measurements. This is a clear advantage of photothermal spectroscopy against absorption spectrophotometry as shown in Fig. 1(b). Photothermal methods are also applicable for strongly scattering samples, which is another advantage against absorption photometry. The non-radiative relaxation process allows the molecules to release the energy of photon as heat and the temperature rise is detected in diverse manners. ${ }^{32-35}$ Typically, the temperature change is observed as a change in the refractive index through optical deflection, lensing, and various interferometric techniques. ${ }^{36-38}$ In photoacoustic spectroscopy, a periodical change in density of the surrounding air is also detected as a sound wave. ${ }^{39}$

\subsection{Principle of TLM}

The general principle of TLS/TLM has already been reviewed, ${ }^{40,41}$ so we will limit the discussion here to just some key points. The total sensitivity of the photothermal detection is augmented by a dimensionless enhancement factor, $E$ described as

$$
E=P_{e}(d n / d T) / \lambda k
$$

where $P_{e}$ is the power of the excitation laser, $\lambda$ is the wavelength of the excitation laser (or probe laser in a dual-beam system), and $k$ is the thermal conductivity of the solvent, containing the molecular properties involved in the conduction of heat. 
Chen, Shimizu, and Kitamori: Review of ultrasensitive readout for micro-/nanofluidic devices...

(1)

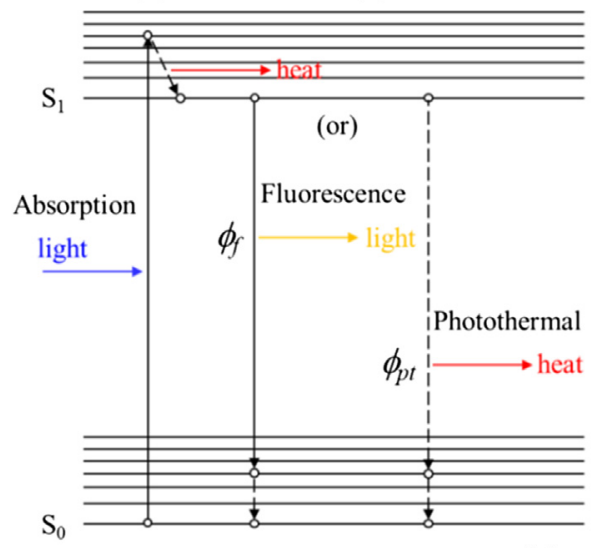

$\phi_{f}, \phi_{p t}:$ Quantum yields

$\phi_{f}+\phi_{p t}=1$

Fluorescent molecule $\phi_{f}:=0.1 \sim 0.5$

$\phi_{p t}:=0.9 \sim 0.5$

Non-fluorescent molecule $\phi_{p t}:=1.0$

(a)

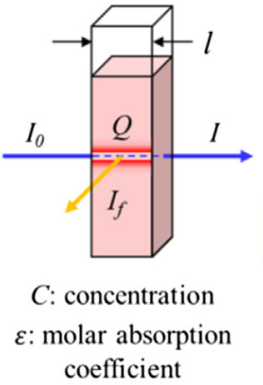

coefficient
Absorbance $\quad \alpha=\varepsilon C l=-\log \frac{I}{I_{o}}$

Fluorescence intensity $\mathrm{I}_{f} \propto \phi_{f}\left(I_{o}-I\right)=\phi_{f} I_{o}\left(1-10^{-\varepsilon C l}\right)$

Photothermal heat $\quad Q \propto \phi_{p t}\left(I_{o}-I\right)=\phi_{p t} I_{o}\left(1-10^{-\varepsilon C l}\right)$

Detectable by TLM

(b)

Fig. 1 Absorption, fluorescence, and photothermal spectroscopy. (a) Jablonski diagram. (b) Expression of the absorption coefficient, fluorescence intensity, and photothermal heat. In absorption spectrophotometry, sensitivity is independent of $I_{0}$, while the fluorescence intensity and photothermal heat are proportional to $I_{0}$. The sample is assumed to be free from scattering and photochemical reactions.

Figure 2 shows optical schemes of single-beam and dual-beam thermal lens experiments. ${ }^{42,43}$ The single-beam instruments, when continuous-wave laser beam incident is used, require timeconsuming averaging and calculation of the thermal lens signal, which response can be quite slow, on the order of a few seconds. It is challenging to measure the temperature-induced signal precisely over this relatively long time scale in the presence of laser instability and fluid convection. In addition, the slow response precludes its usage for continuous online detection. Therefore, dual-beam (excitation and probe beams) thermal lens instruments, which are characterized by their capability of using lock-in detection and providing a sufficiently fast response with a high signal-to-noise ratio, have become widely adopted in analytical practice.

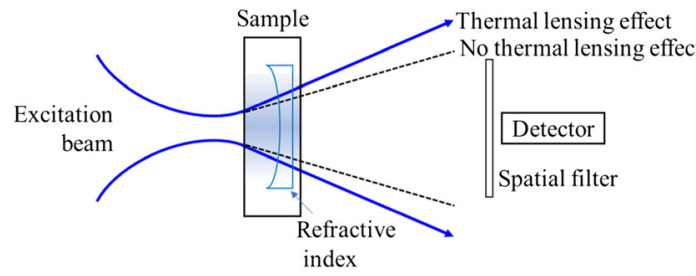

(a)

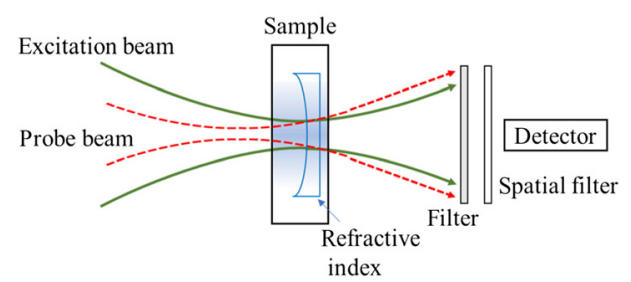

(b)

Fig. 2 Components of thermal lensing experiment. A single-beam setup. The excitation beam generates a temperature-dependent refractive index profile in the sample that can act as a lens. The transmitted light varies with the strength of the lens and is measured using a spatial filter and a detector. (b) A dual-beam setup. Propagation of coaxial probe (dashed line) and excitation (solid line) beams in the thermal lens experiment. The filter prevents the detection of the excitation beam. 


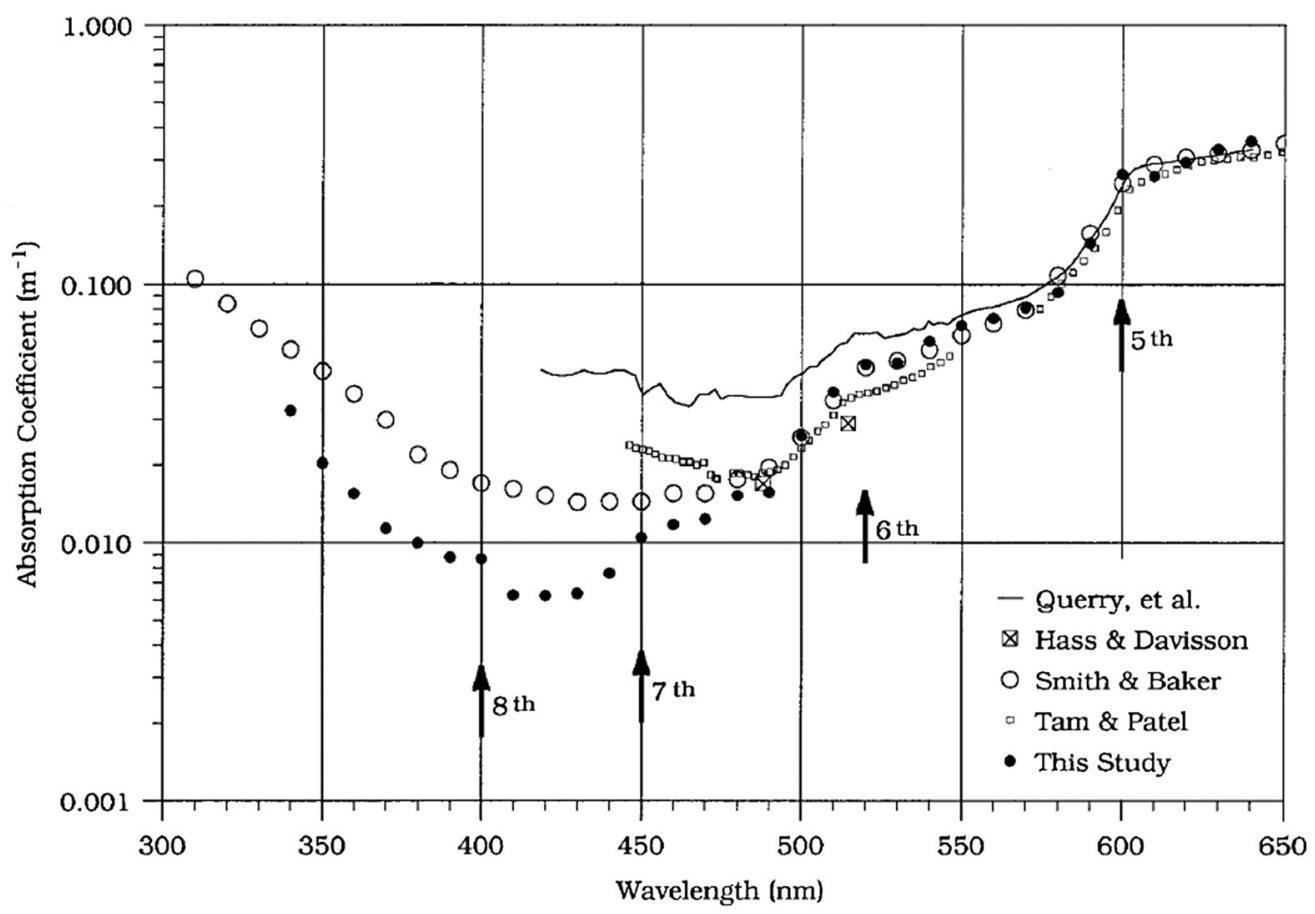

Fig. 3 Absorption spectra of pure water at $25^{\circ} \mathrm{C} .{ }^{44}$ Reproduced with permission, courtesy of The Optical Society.

In the dual-beam TLS, the excitation beam is generally of high power and modulated for increasing the signal-to-noise ratio, while the probe beam is of low power and unmodulated. Ideally, the excitation beam should be absorbed strongly by the analyte to generate the temperature gradient in the sample, while the probe beam has no absorption and is used to generate signals induced by the temperature variation. However, not only the analyte but the solvent may also absorb the energy provided by the lasers and generate a background signal. Hence it is important to choose proper wavelengths for the laser beams and a solvent of desired thermal fluid and optical properties. For instance, water has a v-shaped absorptivity in the VIS and nearUV region with a minimum value of $6.2 \times 10^{-5} \mathrm{~cm}^{-1}$ at $\lambda=420 \mathrm{~nm}$. The absorption becomes more than 50 times stronger $\left(3.31 \times 10^{-3} \mathrm{~cm}^{-1}\right)$ at $\lambda=630 \mathrm{~nm}$ (Fig. 3). ${ }^{44,45}$

\subsection{Instrumentation}

The theory of the thermal lens effect applies to both TLS and TLM. However, compared with TLS, the temperature rise in TLM is usually much smaller, partly due to the higher modulation frequency (e.g., $1 \mathrm{kHz}$ ), the employment of the lower-power excitation laser diode, a shorter (and narrower) optical path, and quicker heat dissipation in a miniaturized system. It is also challenging to conduct the alignment with high reproducibility. In the case of dual-beam (excitation/ probe) photothermal spectrometry, it is hard to overlap the beams without the help of a microscopic alignment system. In addition, the offset between the excitation and probe beam waists is often achieved by incorporating optical components such as beam expanders, which may incur a trade-off between optimized detection sensitivity and instrument complexity. In this section, we summarize recent developments of TLM instruments to tackle these challenges.

\subsubsection{First TLM}

Figure 4(a) shows a schematic of the first TLM system. ${ }^{46}$ The excitation beam is selected to be absorbed by the analyte molecule (in this case, Sunset Yellow FCF, a nonfluorescent dye), but not absorbed by the solvent (water). The probe beam is selected not to be absorbed by the analyte. The excitation beam is chopped at a modulation frequency to generate a periodical 
Chen, Shimizu, and Kitamori: Review of ultrasensitive readout for micro-/nanofluidic devices...

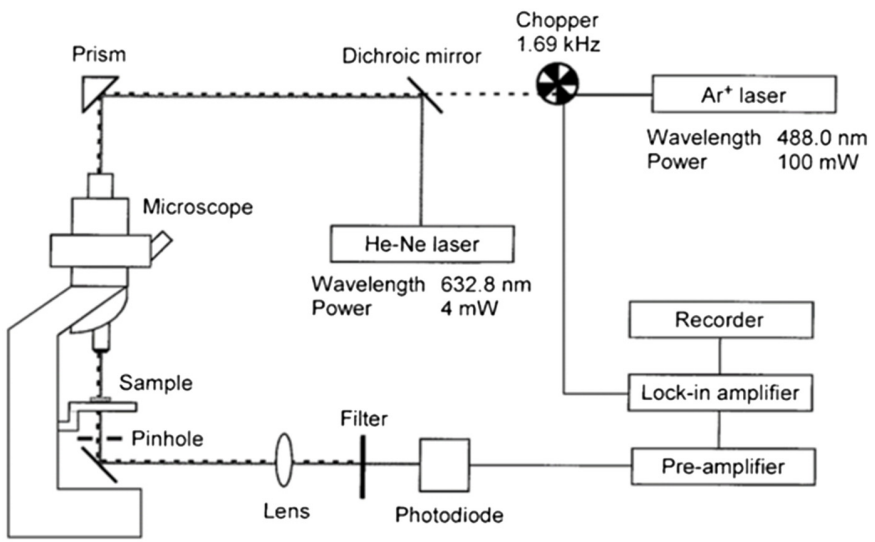

(a)

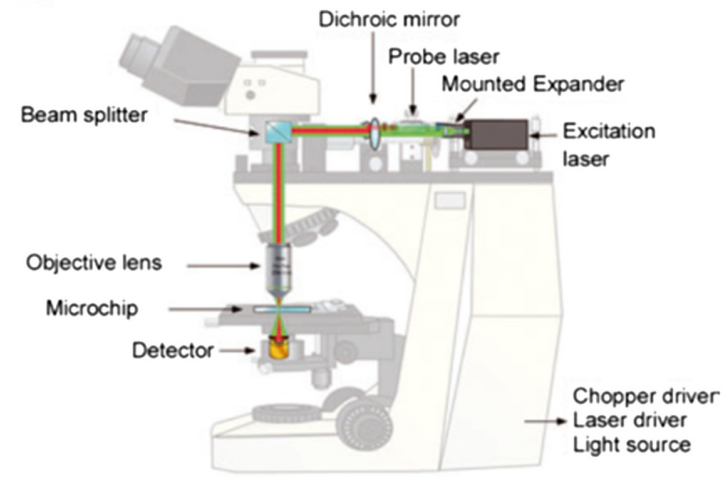

(b)

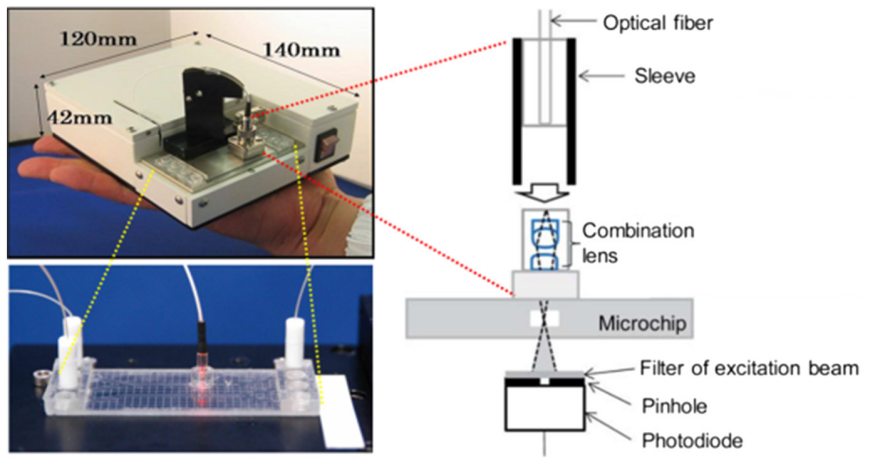

(c)

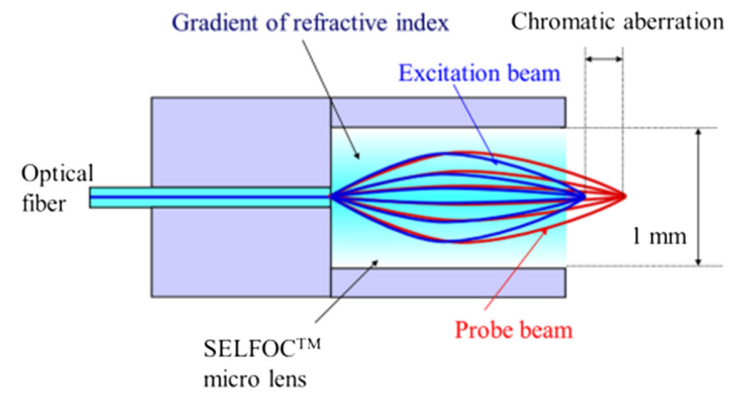

(d)

Fig. 4 Instrumentations of TLM. (a) Schematic of the first TLM. ${ }^{46}$ Reproduced with permission from The Japan Society for Analytical Chemistry. (b) Desk-top TLM. ${ }^{47}$ Reproduced with permission from The Royal Society of Chemistry. (c) Miniaturized TL detection device ${ }^{16}$ and (d) the microlens unit. Adapted with permission from The Royal Society of Chemistry. 
change of temperature in the liquid sample. Hence, when the probe beam is refracted (converged or diverged) by the thermal lens, a periodical change of the probe intensity at the same frequency is detected by the pinhole and the photodiode. The signal from the photodiode and the reference signal from the light chopper are processed using the lock-in amplifier to recover the small AC signal on the large DC probe intensity background. The modulation frequency is typically 1 to $2 \mathrm{kHz}$ for liquid samples and chosen to give the best signal-to-noise ratio. The excitation and probe beams are coaxially introduced into the microscope using a dichroic mirror and tightly focused using an objective lens $(10 \times$ to $40 \times)$. The offset between the excitation and probe beam waists is introduced by exploiting the inherent chromatic aberrations of the objective lens. It is worthy to note that chromatic aberrations of the most modern commercial objective lenses are often too small for the sensitive measurement of the thermal lens. In this paper, a very old microscope with an objective lens that has a large chromatic aberration was used and the offset was nearly at the optimum value by chance.

\subsubsection{Desk-top TLM}

Figure 4(b) shows a desk-top TLM, in which optical components of the first TLM were simplified and installed on the microscope. Here, the offset between the beam waists is achieved by diverging and focusing the collimated excitation beam using the mounted beam expander. ${ }^{47}$ The pinhole before the detector is omitted using a photodiode that has a small aperture. All the optical elements and lasers are assembled on an upright microscope, making the desk-top TLM lightweight and portable. However, laborious and accurate optical alignment between each microchip is still required.

\subsubsection{Thermal lens detection device}

It was later developed by Mawatari et al. ${ }^{16}$ using a small holder consisting of micro-lenses and an optical fiber to focus the collimated laser beams onto the microchip. Only simple attachment and detachment of the optical fiber are needed between each microchip [Fig. 4(c)]. Figure 4(d) shows the focusing of the excitation and probe beams using the micro-lens unit (SELFOC ${ }^{\text {TM}}$ ). ${ }^{48}$ The gradient of the refractive index allowed the beams to be focused on different spots with an optimized chromatic aberration. The authors also assessed the robustness of the TL detection device under flow conditions using nickel(II) phthalocyanine tetrasulfonic acid tetrasodium salt (NiPC) aqueous solutions as a model sample. It was found the flow-induced error was $<2 \%$ when the average flow velocity was below $2 \mathrm{~mm} / \mathrm{s}$ and the excitation beam was modulated at $1.1 \mathrm{kHz}$.

\subsection{Applications}

TLM finds most of its applications in detecting dilute samples due to its superior sensitivity, rapid time response, and remote sensing capability. However, TLM has been striving for low detection limits of analytes in complex solutions, such as biological samples. Also, TLM detects every molecule that has optical absorption at the excitation wavelength, in contrast to laserinduced fluorescence microscopy. As such, additional degrees of specificity (chemical or instrumental) has to been provided. For instance, TLM has been combined with several targeted analyses [e.g., enzyme-linked immunosorbent assay (ELISA) ${ }^{49-52}$ or separation methods (e.g., microchip extraction ${ }^{53-56}$ and liquid chromatography ${ }^{57,58}$ ), delivering instruments with both high sensitivity and selectivity.

Another application of TLM is in the detection of nanoparticles. Current techniques for characterizing nanoparticles include non-optical methods (electron microscopy, atomic force microscopy, and impedance-based detection) and optical methods (nanoparticle tracking analysis, dark-field microscopy, and flow cytometry). There are continuous efforts in achieving the real-time, sensitive, and specific detection of nanoparticles. Most optical methods are either scattering- or fluorescence-based, both of which signals decrease strongly for the small size of nanoparticles. ${ }^{59}$ In contrast, TLM finds its niche in detecting nanoparticles, especially for metallic nanoparticles. ${ }^{60-63}$ Imaging static biological samples have been performed using TLM. ${ }^{64-66}$ TLM may have the potential to detect biological nanoparticles under flow conditions, 
as the detection principle is not directly linked to the size or refraction index of the analyte. However, further improvements on the TLM are imperative to provide the desired sensitivity to discern the small difference of photothermal properties between biological nanoparticles and the surrounding aqueous solution.

\section{TLM at Nanoscale}

\subsection{Principle of Photothermal Optical Phase Shift}

Nanofluidic channels not only provide a further increased surface-to-volume ratio, a comparable fluidic volume to that of a single cell, but also chemical and physical properties deviated from macro- or micro-counterparts. ${ }^{67}$ For instance, the viscosity of water increases 1.4 to 4 times when confined in nanochannels made in silica, which suppresses the diffusion of analytes. ${ }^{68}$ However, as the fluidic channel is down to a submicron thickness, which is comparable to the wavelength of light, the TLM signal level is reduced due to the short sample length and quicker heat dissipation, and the diffraction theory of the thermal lens effect needs to be revisited. ${ }^{69,70}$ Phase-sensitive detections, such as photothermal optical phase shift (POPS) detection, have been exploited to realize background-free detection and to increase the signal-to-background ratio. POPS was first demonstrated as differential interference contrast thermal lens microscopy (DIC-TLM) for the detection of single metallic nanoparticles in microchannels or capillaries, ${ }^{71,72}$ and later further developed for the detection of Sunset Yellow FCF, a nonfluorescent dye in the aqueous solution in nanochannels. ${ }^{73}$ Recently, Maceiczyk et al. ${ }^{74}$ have demonstrated the detection limit of $1.4 \mu \mathrm{M}$ for erythrosin dye in $100 \mathrm{pL}$ droplets at frequencies $>1 \mathrm{kHz}$ using differential detection photothermal interferometry. In POPS shown as an example in Fig. 5, the probe beam is divided into two beams with orthogonal polarizations; one of which is still coaxial with the excitation laser beam, while the other is out of the thermal diffusion zone, providing the reference signal. In this setup, the polarization planes of two probe beams are perpendicular to each other. The thermal lens effect induced by the excitation beam creates the phase contrast between the two probe beams, which can be detected as a new polarization component in the recombined probe beam. Since this principle does not utilize the thermal lens effect, it seems to be more appropriate to call this principle not DIC-TLM but POPS. The radius of the thermal diffusion zone, $\mu$, can be estimated as

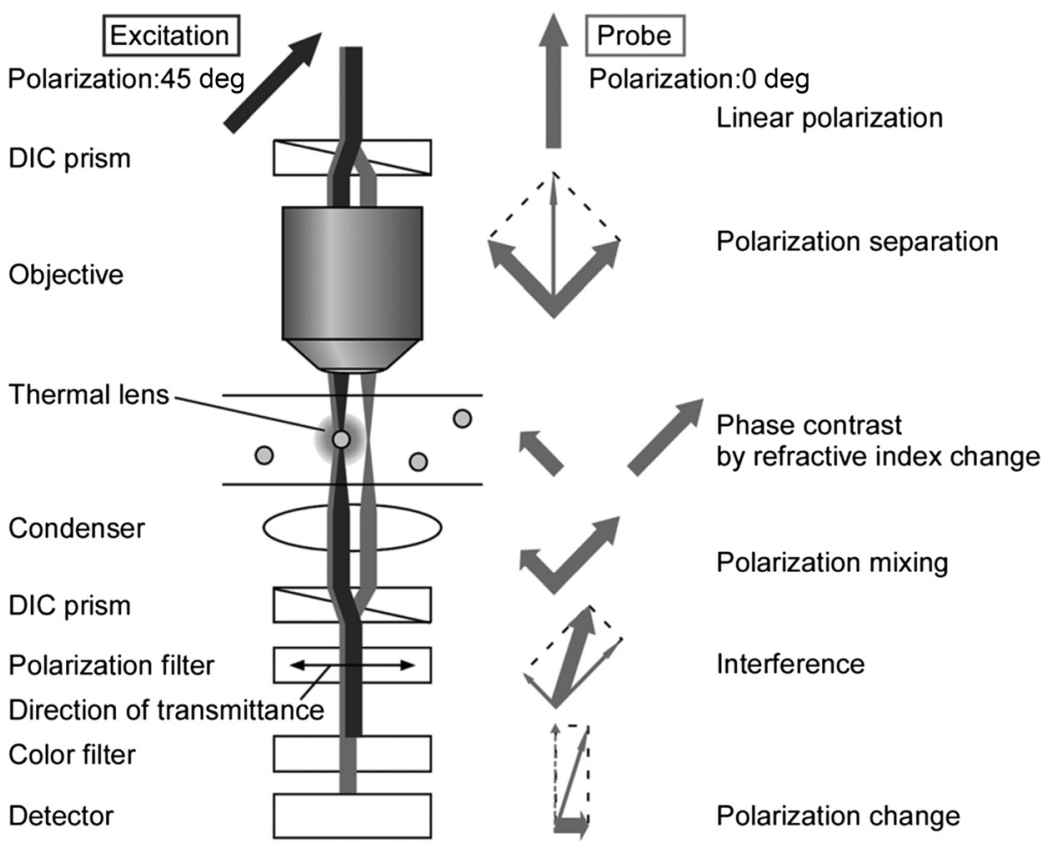

Fig. 5 Principle of POPS. ${ }^{71}$ Reproduced with permission from The Royal Society of Chemistry. 


$$
\mu=\sqrt{\frac{D_{T}}{\pi f}}
$$

where $f$ is the modulation frequency, $D_{T}=k / \rho C_{p}$ is the thermal diffusivity with $k$ is the thermal conductivity, $\rho$ is the density, and $C_{p}$ is the specific heat capacity of the solvent, respectively. Under flow conditions, however, the thermal diffusion zone may no longer be symmetrical and may be exploited to gain advantages. ${ }^{75,76}$ For instance, the reference probe beam should be placed upstream to the actual probe beam to allow for a shorter distance between the two probe beams and a cleaner reference signal. The flow rate may be optimized to minimize the heat transfer from the solvent to the walls of the nanochannel, which can also be done by increasing the modulation frequency at the expense of sensitivity. ${ }^{77}$ This is particularly important for a system in which the temperature gradients $(\mathrm{d} n / \mathrm{d} T)$ of the solvent and chip material are of the opposite polarity. For example, the $\mathrm{d} n / \mathrm{d} T$ of water is $-9.1 \times 10^{-5} \mathrm{~K}^{-1}$, while it is $+9.8 \times 10^{-6} \mathrm{~K}^{-1}$ for fused silica.

\subsection{Instrumentation}

The experimental setup of a POPS detector is shown in Fig. $6{ }^{78}$ In contrast to the setup shown in Fig. 5, the excitation beam is switched from VIS to UV light to detect a wide range of molecules, including proteins, peptides, amino acids, and nucleic acids. The UV excitation laser does not pass through DIC prisms to avoid damaging prisms and to reduce the background signal. It also needs to be considered which optical systems, asymmetric or symmetric, should be adopted. The commercial DIC microscopes are asymmetric systems, which have different objective lens and condenser units. On the illumination side, a Wollaston prism is installed in the condenser unit while a Nomarski prism is used with the objective lens. To produce high contrast, the distance between the two probe beams, which is called shear value, must be greater than the radius of the thermal diffusion zone, $\mu$. Typical DIC prisms designed for biological microscopes have small shear values $(\sim 0.5 \mu \mathrm{m})$ to obtain high-resolution images. Therefore, both the Wollaston and Nomarski prisms must be designed and fabricated specially for TLM, which increases the cost. On the other hand, some DIC prisms designed for metallographic microscopes have relatively larger shear values (several $\mu \mathrm{m}$ ). To use such DIC prisms, the symmetric optical system is suitable, such as the setup shown in Fig. 6.

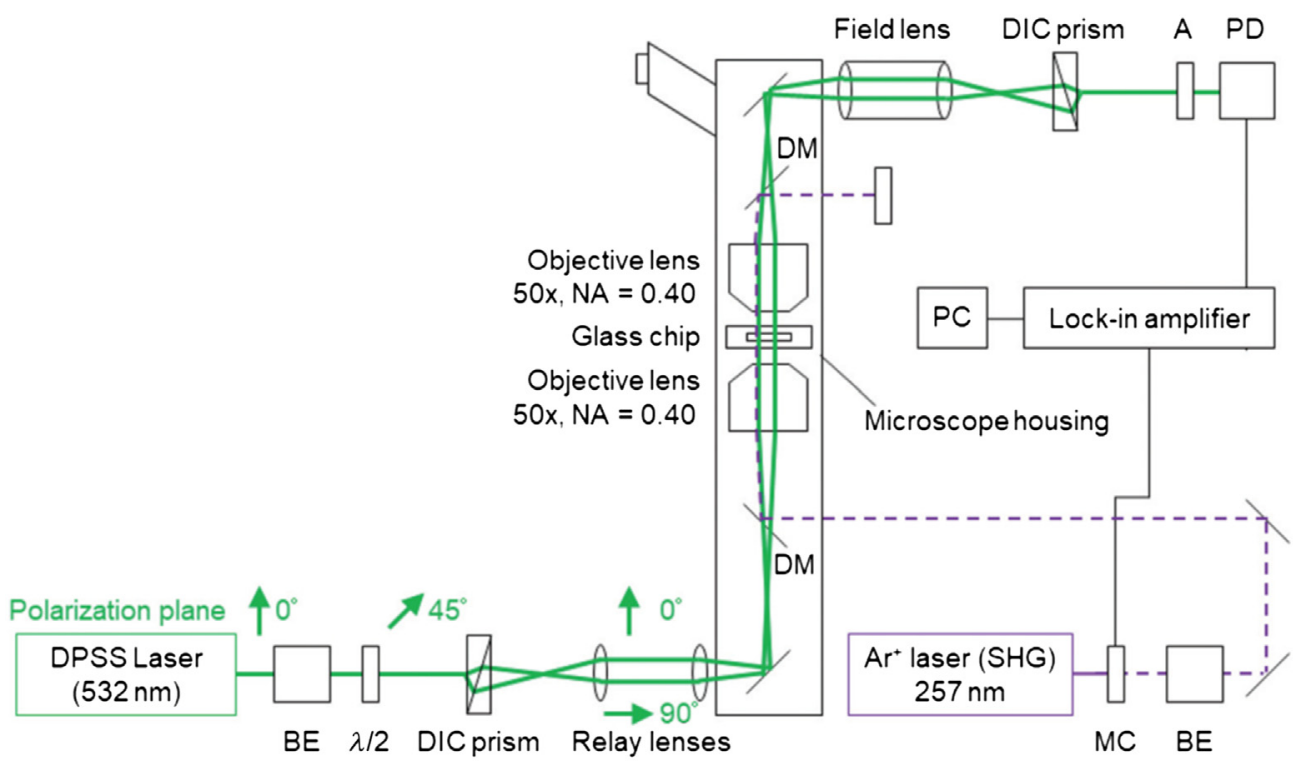

Fig. 6 An instrument of POPS. ${ }^{78}$ Reproduced with permission from The Royal Society of Chemistry. 


\subsection{Applications}

Chromatography is a promising application of TLM at the nanoscale. The extremely high surface-to-volume ratio of nanofluidic channels allows for the rapid separation of molecules by modifying the nanochannel surface. ${ }^{79}$ As well as the highly efficient separation, chemical, and enzymatic reactions, precise liquid manipulation, and sample injection that are necessary for proteomic analyses can be accomplished within several seconds. ${ }^{80}$

The small fluid volume confined in a nanochannel greatly reduces the dilution of the secretome or molecular content of a small number of cells or even a single cell. By performing an on-chip ELISA, interleukin (IL)-6 cytokines secreted by a single B cell were detected at a sensitivity of five molecules. ${ }^{81}$ The utilization of the on-chip ELISA is advantageous since it provides the necessary specificity and the versatility and nearly single-molecule sensitivity. ${ }^{82}$ The same enzymatic reaction can be applied for the detection of various analytes by changing only the recognition molecule. Hence, the TLM system can be used directly without further optimization or characterization, since the same enzymatic product is utilized as the readout.

\section{Perspectives/Concluding Remarks}

The last few decades have witnessed substantial progress in the field of TLM and micro/nanofluidic devices due to technical advances in micro/nanofabrication. Despite these advances, many challenges remain. Currently, single-cell analyses have been the largest targets of applied micro/nanofluidics. ${ }^{83}$ Especially, the high-speed generation and handling of microdroplets contributed to high-throughput analyses including single cell RNA-seq. ${ }^{84,85}$ In this regard, ultrafast cytometry and next-generation sequencing have still been strongly anticipated. ${ }^{86,87}$ Apart from analyses of nucleic acids, single-cell proteomics is clearly a challenge in the next decade. ${ }^{88}$ Since current technologies used in these fields depend on fluorescence, nonlabeled techniques including photothermal spectroscopy are highly expected to simplify the analytical procedures and to reduce the total cost. ${ }^{89,90}$ In basic sciences, it will be important to understand the behavior of molecules confined in nanospace, and the thorough thermal lens theory and simulation at the nanoscale. For the instrument, an electro-optic Kerr cell may be placed in the POPS system to compensate for the phase shift instead of detecting the magnitude of light, which is susceptible to laser light instability. The quest for a widely tunable high-power light source with good beam quality in the UV-VIS region goes on, however, and with such sources, a cheap, robust, and reliable photothermal spectrophotometer applicable to microdevices can be envisaged. ${ }^{91}$ Imaging is also important for biomedical applications of TLM. Not only scanning techniques, but also high-speed, super-resolution, and in vivo imaging should be tackled. ${ }^{92,93}$

\section{Acknowledgments}

This work was supported by the Ministry of Science and Technology of Taiwan (Grant No. MOST 109-2639-E-007-001-ASP). The authors declare no conflicts of interest.

\section{References}

1. T. Kitamori et al., "Thermal lens microscopy and microchip chemistry," Anal. Chem. 76(3), $52 \mathrm{~A}-60 \mathrm{~A}(2004)$.

2. T. Kitamori, "Thermal lens microscope and microchip chemistry," Bull. Chem. Soc. Jpn. 92(2), 469-473 (2019).

3. J. P. Gordon et al., "Long-transient effects in lasers with inserted liquid samples," J. Appl. Phys. 36(1), 3-8 (1965).

4. M. E. Long, R. L. Swofford, and A. C. Albrecht, "Thermal lens technique-new method of absorption spectroscopy," Science 191(4223), 183-185 (1976).

5. N. J. Dovichi and J. M. Harris, "Laser-induced thermal lens effect for calorimetric trace analysis," Anal. Chem. 51(6), 728-731 (1979).

6. A. C. Tam and B. Sullivan, "Remote-sensing applications of pulse photothermal radiometry," Appl. Phys. Lett. 43(4), 333-335 (1983). 
Chen, Shimizu, and Kitamori: Review of ultrasensitive readout for micro-/nanofluidic devices...

7. M. Terazima et al., "Photothermal investigation of the triplet-state of C60," J. Phys. Chem. 95(23), 9080-9085 (1991).

8. K. Uchiyama et al., "Thermal lens microscope," J. Appl. Phys. Part 1 39(Part 1, No. 9A), 5316-5322 (2000).

9. S. Bialkowski, N. G. C. Astrath, and M. A. Proskurnin, Photothermal Spectroscopy Methods, Wiley, Hoboken, NJ (2019).

10. C. H. Chu et al., "Beyond the Debye length in high ionic strength solution: direct protein detection with field-effect transistors (FETs) in human serum," Sci. Rep. 7(1), 5256 (2017).

11. L. J. Xu et al., "Label-free detection of native proteins by surface-enhanced Raman spectroscopy using iodide-modified nanoparticles," Anal. Chem. 86(4), 2238-2245 (2014).

12. F. Vollmer and S. Arnold, "Whispering-gallery-mode biosensing: label-free detection down to single molecules," Nat. Methods 5(7), 591-596 (2008).

13. M. Piliarik and V. Sandoghdar, "Direct optical sensing of single unlabelled proteins and super-resolution imaging of their binding sites," Nat. Commun. 5, 4495 (2014).

14. L. Duan and L. Yobas, "Label-free multiplexed electrical detection of cancer markers on a microchip featuring an integrated fluidic diode nanopore array," ACS Nano 12(8), 7892 7900 (2018).

15. M. Yamauchi et al., "Miniaturized thermal lens and fluorescence detection system for microchemical chips," J. Chromatogr. A 1106(1-2), 89-93 (2006).

16. K. Mawatari, Y. Naganuma, and K. Shimoide, "Portable thermal lens spectrometer with focusing system," Anal. Chem. 77(2), 687-692 (2005).

17. K. Mawatari et al., "Thermal lens detection device," Lab Chip 11(17), 2990-2993 (2011).

18. Y. Kazama and A. Hibara, "Integrated micro-optics for microfluidic detection," Anal. Sci. 32(1), 99-102 (2016).

19. J. Yoosefian and N. Alizadeh, "An optical configuration of crossed-beam photothermal lens spectrometer operating at high flow velocities and its application for cysteine determination in human serum and saliva," Anal. Chem. 90(13), 8227-8233 (2018).

20. M. Tokeshi et al., "Determination of subyoctomole amounts of nonfluorescent molecules using a thermal lens microscope: Subsingle molecule determination," Anal. Chem. 73(9), 2112-2116 (2001).

21. M. Q. Liu, "A flexible thermal lens microscope for highly sensitive detection in microfluidic chips," Laser Phys. Lett. 14(8), 085701 (2017).

22. A. Aota et al., "Microchip-based plasma separation from whole blood via axial migration of blood cells," Anal. Sci. 27(12), 1173-1178 (2011).

23. F. Kitagawa et al., "Toward million-fold sensitivity enhancement by sweeping in capillary electrophoresis combined with thermal lens microscopic detection using an interface chip," J. Chromatogr. A 1106(1-2), 36-42 (2006).

24. K. Sato et al., "Microchip-based immunoassay system with branching multichannels for simultaneous determination of interferon-gamma," Electrophoresis 23(5), 734-739 (2002).

25. T. G. Henares et al., "Multiple enzyme linked immunosorbent assay system on a capillaryassembled microchip integrating valving and immuno-reaction functions," Anal. Chim. Acta 589(2), 173-179 (2007).

26. T. Ohashi et al., "A micro-ELISA system for the rapid and sensitive measurement of total and specific immunoglobulin E and clinical application to allergy diagnosis," Lab Chip 9(7), 991-995 (2009).

27. M. Kakuta et al., "Development of the microchip-based repeatable immunoassay system for clinical diagnosis," Meas. Sci. Technol. 17(12), 3189-3194 (2006).

28. M. Lubej et al., "Microfluidic droplet-based liquid-liquid extraction: online model validation," Lab Chip 15(10), 2233-2239 (2015).

29. M. A. Proskurnin et al., "Advances in thermal lens spectrometry," J. Anal. Chem. 70(3), 249-276 (2015).

30. M. Franko, "Recent applications of thermal lens spectrometry in food analysis and environmental research," Talanta 54(1), 1-13 (2001).

31. S. Adhikari et al., "Photothermal microscopy: imaging the optical absorption of single nanoparticles and single molecules," ACS Nano 14(12), 16414-16445 (2020). 
Chen, Shimizu, and Kitamori: Review of ultrasensitive readout for micro-/nanofluidic devices...

32. S. A. Pfeiffer and S. Nagl, "On-chip photothermal analyte detection using integrated luminescent temperature sensors," Anal. Chem. 89(17), 9400-9406 (2017).

33. P. J. Dennis et al., "Development of a photothermal absorbance detector for use with microfluidic devices," Anal. Chem. 82(10), 4063-4071 (2010).

34. H. G. Chun et al., "Development of a conductivity-based photothermal absorbance detection microchip using polyelectrolytic gel electrodes," J. Chromatogr. A 1523(10), 140-147 (2017).

35. A. W. Tsen et al., "Imaging the electrical conductance of individual carbon nanotubes with photothermal current microscopy," Nat. Nanotechnol. 4(2), 108-113 (2009).

36. W. B. Jackson et al., "Photothermal deflection spectroscopy and detection," Appl. Opt. 20(8), 1333-1344 (1981).

37. A. Harata, H. Nishimura, and T. Sawada, "Laser-induced surface acoustic-waves and photothermal surface gratings generated by crossing two pulsed laser-beams," Appl. Phys. Lett. 57(2), 132-134 (1990).

38. S. Berciaud et al., "Photothermal heterodyne imaging of individual nonfluorescent nanoclusters and nanocrystals," Phys. Rev. Lett. 93(25), 257402 (2004).

39. J. Hodgkinson and R. P. Tatam, "Optical gas sensing: a review," Meas. Sci. Technol. 24(1), 012004 (2013).

40. R. D. Snook and R. D. Lowe, "Thermal lens spectrometry-a review," Analyst 120(8), 2051-2068 (1995).

41. A. Smirnova et al., "Thermooptical detection in microchips: from macro- to micro-scale with enhanced analytical parameters," Electrophoresis 29(13), 2741-2753 (2008).

42. S. J. Sheldon, L. V. Knight, and J. M. Thorne, "Laser-induced thermal lens effect-a new theoretical model," Appl. Opt. 21(9), 1663-1669 (1982).

43. J. Shen, R. D. Lowe, and R. D. Snook, "A model for CW laser induced mode-mismatched dual-beam thermal lens spectrometry," Chem. Phys. 165(2-3), 385-396 (1992).

44. F. M. Sogandares and E. S. Fry, "Absorption spectrum $(340-640 \mathrm{~nm})$ of pure water. 1. Photothermal measurements," Appl. Opt. 36(33), 8699-8709 (1997).

45. S. E. Braslavsky and G. E. Heibel, "Time-resolved photothermal and photoacoustic methods applied to photoinduced processes in solution," Chem. Rev. 92(6), 1381-1410 (1992).

46. K. Sato et al., "Sub-zeptomole detection in a microfabricated glass channel by thermal-lens microscopy," Anal. Sci. 15(6), 525-529 (1999).

47. A. Smirnova et al., "Desktop near-field thermal-lens microscope for thermo-optical detection in microfluidics," Electrophoresis 33(17), 2748-2751 (2012).

48. M. Tokeshi et al., "Thermal lens micro optical systems," Anal. Chem. 77(2), 626-630 (2005).

49. K. Sato et al., "Determination of carcinoembryonic antigen in human sera by integrated bead bed immunoassay in a microchip for cancer diagnosis," Anal. Chem. 73(6), 1213-1218 (2001).

50. M. Ihara et al., "Micro OS-ELISA: rapid noncompetitive detection of a small biomarker peptide by open-sandwich enzyme-linked immunosorbent assay (OS-ELISA) integrated into microfluidic device," Lab Chip 10(1), 92-100 (2010).

51. Y. Y. Dong et al., "Rapid screening swine foot-and-mouth disease virus using micro-ELISA system," Lab Chip 11(13), 2153-2155 (2011).

52. E. Mori et al., "A sensitive and rapid assay of BNP in patient blood by micro-ELISA," Anal. Methods 9(19), 2830-2834 (2017).

53. H. Hisamoto et al., "On-chip integration of sequential ion-sensing system based on intermittent reagent pumping and formation of two-layer flow," Anal. Chem. 73(22), 5551-5556 (2001).

54. H. Hisamoto et al., "On-chip integration of neutral ionophore-based ion pair extraction reaction," Anal. Chem. 73(6), 1382-1386 (2001).

55. T. Minagawa, M. Tokeshi, and T. Kitamori, "Integration of a wet analysis system on a glass chip: determination of $\mathrm{Co}$ (II) as 2-nitroso-1-naphthol chelates by solvent extraction and thermal lens microscopy," Lab Chip 1(1), 72-75 (2001).

56. A. Abbasi-Ahd, N. Shokoufi, and K. Kargosha, "Headspace single-drop microextraction coupled to microchip-photothermal lens microscopy for highly sensitive determination of captopril in human serum and pharmaceuticals," Microchim. Acta 184(7), 2403-2409 (2017). 
Chen, Shimizu, and Kitamori: Review of ultrasensitive readout for micro-/nanofluidic devices...

57. S. Hiki et al., "UV excitation thermal lens microscope for sensitive and nonlabeled detection of nonfluorescent molecules," Anal. Chem. 78(8), 2859-2863 (2006).

58. M. Isoda, M. Fukuma, and A. Harata, "Ultraviolet-excitation photothermal heterodyne interferometer as a micro-HPLC detector," Anal. Sci. 35(12), 1311-1315 (2019).

59. C. Y. Chiang and C. C. Chen, "Toward characterizing extracellular vesicles at a singleparticle level," J. Biomed. Sci. 26, 9 (2019).

60. K. Mawatari, T. Kitamori, and T. Sawada, "Individual detection of single nanometer-sized particles in liquid by photothermal microscope," Anal. Chem. 70(23), 5037-5041 (1998).

61. D. Boyer et al., "Photothermal imaging of nanometer-sized metal particles among scatterers," Science 297(5584), 1160-1163 (2002).

62. S. Yamaoka et al., "Efficient thermal lens nanoparticle detection in a flow-focusing microfluidic device," Sens. Actuators B 228, 581-586 (2016).

63. N. Shokoufi, B. A. N. Asbaghi, and A. Abbasi-Ahd, "Microfluidic chip-photothermal lens microscopy for DNA hybridization assay using gold nanoparticles," Anal. Bioanal. Chem. 411(23), 6119-6128 (2019).

64. M. V. Khodakovskaya et al., "Complex genetic, photothermal, and photoacoustic analysis of nanoparticle-plant interactions," Proc. Natl. Acad. Sci. U.S.A. 108(3), 1028-1033 (2011).

65. D. A. Nedosekin et al., "Photoacoustic and photothermal detection of circulating tumor cells, bacteria and nanoparticles in cerebrospinal fluid in vivo and ex vivo," J. Biophotonics 6(6-7), 523-533 (2013).

66. Z. A. Nima et al., "Bioinspired magnetic nanoparticles as multimodal photoacoustic, photothermal and photomechanical contrast agents," Sci. Rep. 9, 887 (2019).

67. T. Tsukahara et al., "NMR study of water molecules confined in extended nanospaces," Angew. Chem. Int. Ed. 46(7), 1180-1183 (2007).

68. A. Hibara et al., "Nanochannels on a fused-silica microchip and liquid properties investigation by time-resolved fluorescence measurements," Anal. Chem. 74(24), 6170-6176 (2002).

69. Y. Tsuyama, K. Morikawa, and K. Mawatari, "Nanochannel chromatography and photothermal optical diffraction: femtoliter sample separation and label-free zeptomole detection," J. Chromatogr. A 1624, 461265 (2020).

70. Y. Tsuyama and K. Mawatari, "Nonfluorescent molecule detection in 10(2) nm nanofluidic channels by photothermal optical diffraction," Anal. Chem. 91(15), 9741-9746 (2019).

71. H. Shimizu, K. Mawatari, and T. Kitamori, "Development of a differential interference contrast thermal lens microscope for sensitive individual nanoparticle detection in liquid," Anal. Chem. 81(23), 9802-9806 (2009).

72. R. Maceiczyk et al., "A photothermal spectrometer for fast and background-free detection of individual nanoparticles in flow," Anal. Chem. 89(3), 1994-1999 (2017).

73. H. Shimizu, K. Mawatari, and T. Kitamori, "Sensitive determination of concentration of nonfluorescent species in an extended-nano channel by differential interference contrast thermal lens microscope," Anal. Chem. 82(17), 7479-7484 (2010).

74. R. M. Maceiczyk et al., "Differential detection photothermal spectroscopy: towards ultrafast and sensitive label-free detection in picoliter \& femtoliter droplets," Lab Chip 17(21), 3654-3663 (2017).

75. Y. Kikutani et al., "Flowing thermal lens micro-flow velocimeter," Sens. Actuators B 133(1), 91-96 (2008).

76. M. Q. Liu, S. Malovrh, and M. Franko, "Microfluidic flow-injection thermal-lens microscopy for high-throughput and sensitive analysis of sub- $\mu$ L samples," Anal. Methods 8(25), 5053-5060 (2016).

77. H. Shimizu et al., "Thermo-optical characterization of photothermal optical phase shift detection in extended-nano channels and UV detection of biomolecules," Anal. Chem. 89(11), 6043-6049 (2017).

78. H. Shimizu et al., "Ultrasensitive detection of nonlabelled bovine serum albumin using photothermal optical phase shift detection with UV excitation," Analyst 145(7), 2580-2585 (2020).

79. A. Smirnova et al., "Reversed-phase chromatography in extended-nano space for the separation of amino acids," J. Chromatogr. A 1418, 224-227 (2015). 
Chen, Shimizu, and Kitamori: Review of ultrasensitive readout for micro-/nanofluidic devices...

80. H. Shimizu et al., "Extended-nano chromatography," J. Chromatogr. A 1490, 11-20 (2017).

81. T. Nakao et al., "Cytokine analysis on a countable number of molecules from living single cells on nanofluidic devices," Analyst 144(24), 7200-7208 (2019).

82. K. Shirai et al., "A single-molecule ELISA device utilizing nanofluidics," Analyst 143(4), 943-948 (2018).

83. T. W. Murphy et al., "Recent advances in the use of microfluidic technologies for single cell analysis," Analyst 143(1), 60-80 (2018).

84. L. R. Shang, Y. Cheng, and Y. J. Zhao, "Emerging droplet microfluidics," Chem. Rev. 117(12), 7964-8040 (2017).

85. M. Singh et al., "High-throughput targeted long-read single cell sequencing reveals the clonal and transcriptional landscape of lymphocytes," Nat. Commun. 10, 3120 (2019).

86. N. Nitta et al., "Intelligent image-activated cell sorting," Cell 175(1), 266-276.e13 (2018).

87. M. J. P. Chaisson et al., "Resolving the complexity of the human genome using singlemolecule sequencing," Nature 517(7536), 608-611 (2015).

88. Y. F. Liu et al., "Advancing single-cell proteomics and metabolomics with microfluidic technologies," Analyst 144(3), 846-858 (2019).

89. Y. Suzuki et al., "Label-free chemical imaging flow cytometry by high-speed multicolor stimulated Raman scattering," Proc. Natl. Acad. Sci. U.S.A. 116(32), 15842-15848 (2019).

90. V. P. Zharov, E. I. Galanzha, and V. V. Tuchin, "Photothermal flow cytometry in vitro for detection and imaging of individual moving cells," Cytom. Part A 71A(4), 191-206 (2007).

91. H. Cabrera et al., "Trace detection and photothermal spectral characterization by a tuneable thermal lens spectrometer with white-light excitation," Talanta 183, 158-163 (2018).

92. M. Tamamitsu et al., "Quantitative phase imaging with molecular vibrational sensitivity," Opt. Lett. 44(15), 3729-3732 (2019).

93. Z. M. Li et al., "Super-resolution far-field infrared imaging by photothermal heterodyne imaging," J. Phys. Chem. B 121(37), 8838-8846 (2017).

Chihchen Chen is an associate professor of the Institute of NanoEngineering and MicroSystems, Department of Power Mechanical Engineering at the National Tsing Hua University, Hsinchu, Taiwan. She received her dual $\mathrm{PhD}$ in bioengineering and nanotechnology from the University of Washington in 2006. Her current research interests include micro-/ nanofluidic and optical detection systems, with a focus on the isolation and characterization of the cellular and sub-cellular components.

Hisashi Shimizu is a project assistant professor at The University of Tokyo. He received his BS, MS, and PhD degrees in engineering from The University of Tokyo in 2007, 2009, and 2012, respectively. His current research interests include photothermal spectroscopy, micro/nanofluidics, and separation chemistry. He is a member of the Chemical Society of Japan, the Japan Society for Analytical Chemistry, and the Society for Chemistry and Micro-Nano Systems.

Takehiko Kitamori is honorary chair professor at the National Tsing Hua University, Taiwan, honorary visiting professor at Lund University, Sweden, and project professor at The University of Tokyo, Japan. He worked for Hitachi Ltd. after graduation from The University of Tokyo, and received a $\mathrm{PhD}$ in 1989. He pioneered micro/nanofluidics, and his current research interest includes its enhancement to social implementation. He is honorary doctor at Lund University, and Foreign Member of Royal Swedish Academy of Science. 